\title{
Cutaneous Tuberculosis and AIDS Diagnosis: A Case Report
}

\author{
Gisela Borges*, Maria de Jesus Silva \\ Internal Medicine Department, Hospital Pulido Valente, Lisbon, Portugal \\ Email: * giselatavborges@gmail.com
}

How to cite this paper: Borges, G. and de Jesus Silva, M. (2018) Cutaneous Tuberculosis and AIDS Diagnosis: A Case Report. Case Reports in Clinical Medicine, 7, 126130.

https://doi.org/10.4236/crcm.2018.72010

Received: January 14, 2018

Accepted: February 10, 2018

Published: February 13, 2018

Copyright (c) 2018 by authors and Scientific Research Publishing Inc. This work is licensed under the Creative Commons Attribution International License (CC BY 4.0).

http://creativecommons.org/licenses/by/4.0/

\begin{abstract}
The incidence of extrapulmonary tuberculosis has been increasing especially among immunocompromised patients. Mycobacterium tuberculosis skin infection is rare, accounting for $1.5 \%$ of all forms of the disease. We present a clinical case of 38 years old woman, Brazilian; with sexual risk behaviours that develops a sudden thoracoabdominal tumefaction of $4-5 \mathrm{~cm}$. The investigation confirmed a metastatic tuberculous abscess in a patient with acquired immunodeficiency syndrome.
\end{abstract}

\section{Keywords}

Cutaneous Tuberculosis, Metastatic Tuberculous Abscess, Tuberculous Gumma, Extrapulmonary Tuberculosis, Acquired Immunodeficiency Syndrome

\section{Introduction}

Tuberculosis (TB) is caused by bacillus Mycobacterium tuberculosis, more than two billion people (about one-third of the world population) are estimated to be affected [1]. The re-emergence of this infectious disease at this century has been associated with HIV infection, the use of immunosuppressive agents and appearance of multidrug-resistance.

Most of tuberculosis cases have a pulmonary manifestation; nevertheless the Koch's bacillus can be disseminated haematogenously leading to multiplication in other organs and systems [2]. Cutaneous tuberculosis (CTB) is a rare form of extrapulmonary involvement and may arise secondary to lung disease or by direct inoculation of the bacillus on the skin. It represents $1.5 \%$ of all forms of disease [3], accounting for $0.04 \%$ to $2 \%$ of all dermatological conditions [4].

Among HIV positive patients, TB is 9 to 16 times higher [5] and the clinical 
features may be atypical [6]. Opportunistic infections, like CTB are more prone to appear when the patients are severely immunocompromised (lymphocytes $\mathrm{T}$ CD4 $<200$ cells $/ \mathrm{mm}^{3}$ and very high load RNA-HIV); according to Centers for Disease Control and Prevention (CDC) they are classified to have Acquired Immuno Deficiency Syndrome (AIDS).

The case in discussion highlights the importance of excluding CTB in imunocompromised patients presenting skin lesions.

\section{Case Report}

We present a clinical case of a 38 years old Brazilian woman, a beautician, with a history of sexual risk behaviours in the past and currently, who was admitted in our medicine department for investigation of a sudden onset thoracoabdominal tumefaction. She also referred asthenia, nausea, anorexia and a weight loss of about $4 \mathrm{~kg}$ during the last two months. She had been medicated with amoxicillin/clavulanate for an acute tracheobronchitis 20 days before the actual disease. On the physical examination, there was a tumefaction of 4 to $5 \mathrm{~cm}$ on the transition of left hemithorax and hypochondrium, tender and painful with no signs of fluctuation; with regular and well defined contours (Figure 1).

An abdominal ultrasound was performed followed by Computed Tomography scan (CT scan) evidencing a semi-liquid collection of $40-54 \mathrm{~mm}$ with its origin at the intercostal space-probable abscess (Figure 2(a), Figure 2(b)), and cylindrical bronchiectasis on the left posterior basal region of pulmonary parenchyma.

The abscess was drained and mycobacteriological culture of the purulent content revealed non-resistant Mycobacterium tuberculosis complex. HIV serology revealed positive for HIV subtype 1, with viral load of 1,720,000 copies/mL (6.24 Log) and $115 \mathrm{CD} 4 \mathrm{~T}$ lymphocyte cell count.

The final diagnostic was extrapulmonary tuberculosis in a patient with AIDS criteria, according to the CDC classification. First line tuberculostatic therapy was initiated (isoniazid $300 \mathrm{mg}$, pyrazinamide $1000 \mathrm{mg}$, rifampicin $450 \mathrm{mg}$ and etambutol $800 \mathrm{mg}$ ) and a few days later antiretroviral. There was a complete resolution of the skin lesion and a good virological control of the HIV infection.

\section{Discussion}

Several mycobacterias (M. tuberculosis, M. bovis, M. africanum, M. microti, $M$. canetti, $M$. caprae, among others) may be involved in cutaneous diseases, but the most frequent is M. tuberculosis. Vaccination with intradermal injection of the bacille Calmette-Guérin (BCG) has been also associated to skin lesions. In most cases, tuberculosis is transmitted by air but rarely cutaneous manifestations can occur. The risk of developing disease increases in the presence of HIV infection, intravenous drug users, diabetes mellitus, immunosuppressive therapy, malignancy, end-stage renal disease or lactation [7]. 


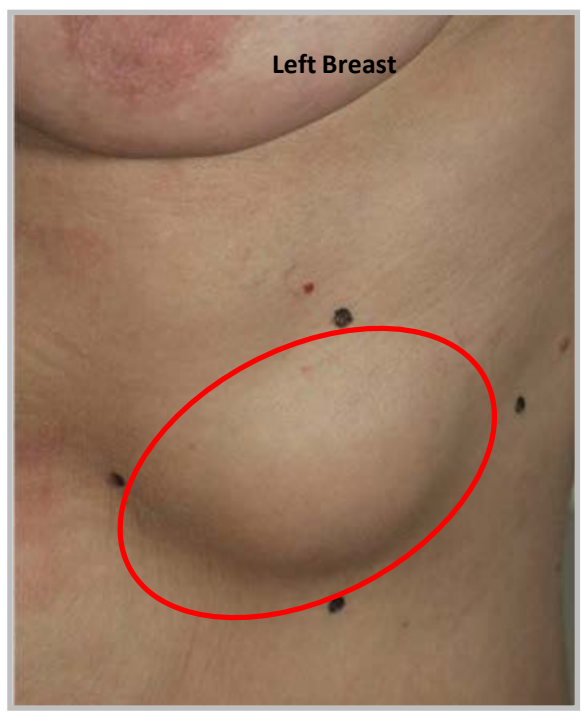

Figure 1. Initial presentation of the thoracoabdominal tumefaction.

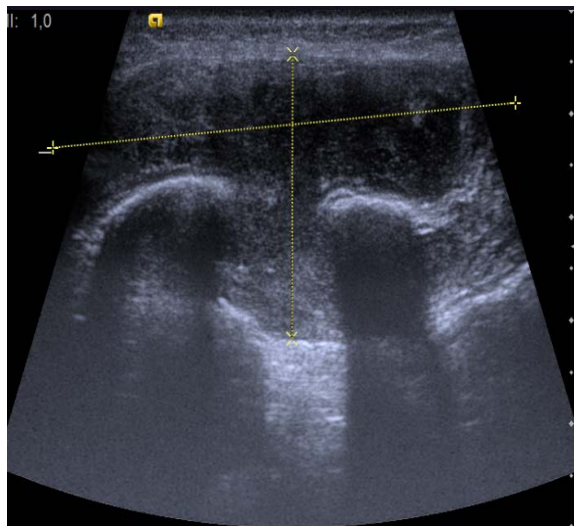

(a)

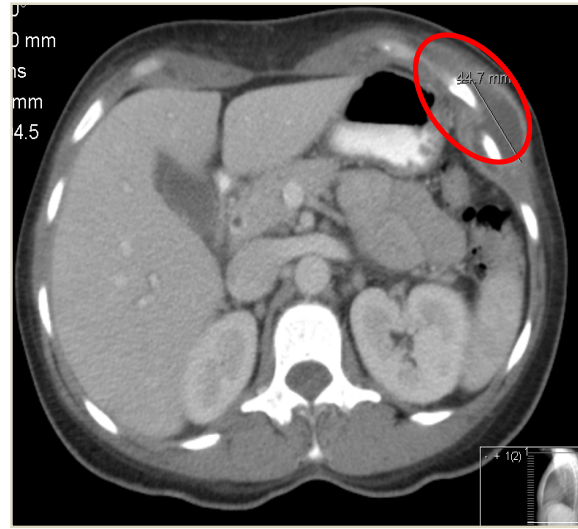

(b)

Figure 2. Abdominal Ultrasound and Computed Tomography scan respectively, revealing an abscess of the thoracoabdominal wall.

Tuberculous gumma is a multibacillary form of TB that can occur even without a previous source of contact. TB and particularly CTB are unusual in industrialized countries, and most cases happen in developing countries. Some retrospective studies developed by Terranova et al., in north Ethiopia (2007) and Assane et al., in Dakar (2010) described $8.9 \%$ and $11.25 \%$ cases of tuberculous gumma, respectively [8] [9]. In Terranova et al. study, $22 \%$ of all patients were infected by HIV [8].

Tuberculous gumma is usually single or multiple nontender, fluctuant nodules develop forming draining sinus abscesses unless surgically incised and drained. No predominance concerning the location of the infection has been described. According to an epidemiological study-Umapathy et al.-in two major teaching Hospitals in India (Chennai), extremities were the predominant location affected [6]. 
Other causes of skin lesion should be excluded as staphylococcal abscess, other mixed bacterial infections, sporotrichosis, nocardiosis, chromomycosis, leishmaniosis, atypical mycobacterial infections, deep fungal infections, syphilitic gumma, leprosy and all forms of panniculitis [10].

In the reported case, the diagnosis tuberculous gumma was established after isolation of Mycobacterium tuberculosis in the pus. The immunosupression due to an advanced HIV infection probably contributed to the onset and evolution of the disease.

The bacillary load in CTB is much lower than in pulmonary tuberculosis, with great response to first line tuberculostatic drugs (two months of quadruple therapy-isoniazid, rifampicin, pyrazinamide and ethambutol, followed by four months of double therapy-isoniazid plus rifampicin) [11]. When obtaining a TB (or CTB) diagnosis it is mandatory to investigate patient's immunological status, habits and epidemiologic factors. HIV patients with history of tuberculosis present higher risk of resistant strains.

In the presented case, the mycobacteriological culture obtained an isolation of Mycobacterium tuberculosis complex, sensitive to all first line tuberculostatics. We verified a complete resolution of the cutaneous lesion without any sequelae or scar. Surgical excision might be necessary, adjuvant to pharmacological therapy but also as a diagnostic method in order to obtain a cultural result. The fact of our patient referring sexual risk behaviour, combined with a prolonged consumptive condition alerted us to investigate the presence of HIV infection. The serology came positive for HIV infection subtype 1, presenting an advanced immunosupression with clinical AIDS disease (lymphocytes T CD $4<200$ cells and extrapulmonary tuberculosis). CTB should be part of the differential diagnosis of skin lesions in patients with HIV infection and must be excluded, and even more if there is a history of pulmonary tuberculosis.

\section{References}

[1] Lönnroth, K. and Raviglione, M. (2008) Global Epidemiology of Tuberculosis: Prospects for Control. Seminars in Respiratory and Critical Care Medicine, 29, 481. https://doi.org/10.1055/s-0028-1085700

[2] Almaguer-Chávez, J., Ocampo-Candiani, J. and Rendón, A. (2009) Current Panorama in the Diagnosis of Cutaneous Tuberculosis. Actas Dermo-Sifiliográficas, 100, 562-570. https://doi.org/10.1016/S0001-7310(09)71904-0

[3] Farina, M.C., Gegundez, M.I. and Pique, E. (1995) Cutaneous Tuberculosis: A Clinical, Histopathologic and Bacteriologic Study. Journal of the American Academy of Dermatology, 33, 433-440. https://doi.org/10.1016/0190-9622(95)91389-0

[4] Umapathy, K.C., Begum, R., Ravichandran, G., Rahman, F., Paramasivan, C.N. and Ramanathan, V.D. (2006) Comprehensive Findings on Clinical, Bacteriological, Histopathological and Therapeutic Aspects of Cutaneous TUBERCULOSIS. Tropical Medicine \& International Health, 11, 1521-1528. https://doi.org/10.1111/j.1365-3156.2006.01705.x

[5] Antonucci, G., Girardi, E., Raviglione, M.C. and Ippolito, G. (1995) Risk Factors for Tuberculosis in HIV-Infected Persons. A Prospective Cohort Study. The Gruppo 
Italiano di Studio Tubercolosi e AIDS (GISTA). JAMA, 274, 143.

https://doi.org/10.1001/jama.1995.03530020061033

[6] Chaisson, R.E. and Slutkin, G. (1989) Tuberculosis and Human Immunodeficiency Virus Infection. The Journal of Infectious Diseases, 159, 96-100. https://doi.org/10.1093/infdis/159.1.96

[7] Frankel, A., Penrose, C. and Emer, J. (2009) Cutaneous Tuberculosis, a Practical Case Report and Review for the Dermatologist. The Journal of Clinical and Aesthetic Dermatology, 2, 19-27.

[8] Teranova, M., Padovese, V., Fornari, U. and Morrone, A. (2008) Clinical and Epidemiological Study of Cutaneous Tuberculosis in Northern Ethiopia. Dermatology, 217, 89-93. https://doi.org/10.1159/000128284

[9] Assane, K., Oumou, N.S., Mohamed, C., Ndiaye, S.T., Moussa, D., Thierno, D.M., et al. (2010) Cutaneous Tuberculosis in Dakar: 151 Cases Report. Mali Medical, 25, 14-17.

[10] Ghosh, S., Aggarwal, K., Jain, V.K., Chauduri, S., Ghosh, E. and Arshdeep (2014) Tuberculosis Verrucosa Cutis Presenting as Diffuse Plantar Keratoderma: An Unusual Sight. Indian Journal of Dermatology, 59, 80-81.

[11] Bravo, F.G. and Gotuzzo, E. (2007) Cutaneous Tuberculosis. Clinics in Dermatolo$g y$, 25, 173-180. https://doi.org/10.1016/j.clindermatol.2006.05.005 